\title{
Markovian approach to tackle the interaction of simultaneous diseases
}

\author{
D. Soriano-Paños $\odot,{ }^{1,2}$ F. Ghanbarnejad, ${ }^{3,4,5}$ S. Meloni $\odot,{ }^{6}$ and J. Gómez-Gardeñes $\oplus^{1,2, *}$ \\ ${ }^{1}$ GOTHAM Laboratory, Institute for Biocomputation and Physics of Complex Systems (BIFI), University of Zaragoza, 50018 Zaragoza, Spain \\ ${ }^{2}$ Departamento de Física de la Materia Condensada, Universidad de Zaragoza, 50009 Zaragoza, Spain \\ ${ }^{3}$ Institute of Theoretical Physics, Technical University of Berlin, Hardenbergstr. 36, Sekr. EW 7-1 D-10623 Berlin \\ ${ }^{4}$ Quantitative Life Sciences (QLS), The Abdus Salam International Centre for Theoretical Physics (ICTP), 34151 Trieste, Italy \\ ${ }^{5}$ Department of Physics, Sharif University of Technology, P.O. Box 11165-9161, Tehran, Iran \\ ${ }^{6}$ IFISC, Instituto de Física Interdisciplinar y Sistemas Complejos (CSIC-UIB), 07122 Palma de Mallorca, Spain
}

(Received 10 July 2019; published 19 December 2019)

\begin{abstract}
The simultaneous emergence of several abrupt disease outbreaks or the extinction of some serotypes of multistrain diseases are fingerprints of the interaction between pathogens spreading within the same population. Here, we propose a general and versatile benchmark to address the unfolding of both cooperative and competitive interacting diseases. We characterize the explosive transitions between the disease-free and the epidemic regimes arising from the cooperation between pathogens and show the critical degree of cooperation needed for the onset of such abrupt transitions. For the competing diseases, we characterize the mutually exclusive case and derive analytically the transition point between the full-dominance phase, in which only one pathogen propagates, and the coexistence regime. Finally, we use this framework to analyze the behavior of the former transition point as the competition between pathogens is relaxed.
\end{abstract}

DOI: 10.1103/PhysRevE.100.062308

\section{INTRODUCTION}

Containing the spread of infectious diseases constitutes one of the major challenges of modern societies [1]. During the past decades, empowered with tools from nonequilibrium statistical physics and nonlinear dynamics, classical epidemic models [2-5] have been progressively refined to capture the many ingredients that interplay to give rise to real epidemic outbreaks [6-9]. Epidemic modeling was particularly boosted by the incorporation of complex interaction networks [10-12]: Capturing the backbone of interactions through which pathogens spread allowed us to study how network heterogeneity influences epidemic onsets [13-16].

The advances made in the representation of the interaction map in a variety of complex systems (such as multilayer frameworks [17-19] or time-varying graphs [20]) have allowed us to improve epidemic models to tackle the analysis about the role played by human interactions in contagion processes. Examples of the ingredients incorporated in epidemic models include: the volatile nature of human contacts [21-24], the multiscale and recurrent mobility patterns in metapopulation models [25-29], the coexistence of multiple interaction or mobility modes [30-32], and the adaptation of human behavior to epidemic waves [33-36]. All these studies put the focus on understanding the role of the former ingredients to propose efficient containment policies [37-39] aimed at controlling and preventing the unfolding of epidemic states.

The vast majority of these models are designed to characterize the spreading dynamics of single pathogens whose evolution is assumed not to depend on the presence of others.

\footnotetext{
*gardenes@unizar.es
}

However, many diseases do not fulfill this assumption, since their spreading patterns are strongly influenced by the simultaneous propagation of other pathogens. One paradigmatic example where the interaction between simultaneous diseases played a crucial role on their impact took place in 1918, manifested by a sudden abrupt increase in the death rate per Pneumonia cases matched with the onset of the Spanish Flu [40]. The correlation between both diseases clearly suggested their interplay in a cooperative way. Another emblematic case is the interaction between Tuberculosis (TB) and HIV. WHO reports [41] that the estimated risk of developing TB is 16-27 times higher in subjects with HIV than in the normal population and that of the 10.4 million estimated cases of TB worldwide, 1.2 million (the $11 \%$ of the total) are among people living with HIV. However, there are other examples in which the presence of one pathogen is detrimental to the propagation of other infections, since being infected by one disease confers partial or total immunity with respect to the other one. Competitive interaction between diseases typically occurs within the different serotypes of multistrain diseases, such as DENV [42] and influenza [44], but also takes place between pathogens corresponding to different diseases, such as the recently reported interaction between ZIKV and DENV [43]. For Influenza, for example, it has been proved that interaction between strains in one season shapes the evolution of the virus and development of the following season and that the cross-immunity between strains-i.e., immunity to one strain obtained through the exposure to a similar strain-from one season to the other can reach up to the $88 \%$ [45].

In recent years, several works [46-57] have tackled the extension of epidemic models to introduce the interaction between different coexisting diseases (see Ref. [58] for a recent review on the subject). Remarkably, interesting theoretical 
results have been already found such as the emergence of first-order transitions when two diseases cooperate [46-52] or the extinction of some infectious strains due to the competition with their counterparts [53-57]. However, some of these findings have been obtained at the expense of relying on strong assumptions regarding the contagion network structure or the mechanisms driving the interaction between pathogens, thus limiting their application to real scenarios where cooperative or competitive spread of pathogens occurs. In addition, many of the current models are specifically focused on understanding the effects of introducing either strong cooperation or mutually exclusive competition between two diseases. This way, the formulation of a consistent mathematical framework to characterize diseases whose interaction lies in between the former extreme cases remains an open theoretical challenge.

In this work, we propose a general epidemic model for characterizing the spread of interplaying pathogens with arbitrary degree of interaction, i.e., ranging from the mutually exclusive case to the strong cooperative regime. This new framework is constructed by following the Microscopic Markov Chain Approach (MMCA) [14,59]. This formalism enables us to keep all the information about the structure of the contact network and to track the evolution of individual nodes, i.e., without the need of statistical assumptions such as considering that all the agents within the same degree class are dynamically equivalent (as assumed in the heterogeneous mean field approximation). We show that the Markovian equations here proposed reproduce with great accuracy the variety of phenomena observed in Monte Carlo simulations, such as the explosive epidemic outbreaks in the cooperative case, thus providing an alternative to computationally expensive simulations. Despite the great agreement observed in most of the phase diagram, some slight discrepancies appear close to the critical points due to the emergence of strong dynamical correlations induced by the contact network. The accuracy of the Markovian framework opens the door to a reliable mathematical analysis of the model. As an example, we derive analytically the second epidemic threshold that, in the fullycompetitive case, separates the regions of full-dominance by one single pathogen and that of coexistence.

The manuscript is organized as follows. In Sec. II we present the description of the model for interacting diseases and explain the mathematical formalism, including the theoretical assumptions and the rationale behind the equations governing the evolution of the system. In Secs. III and IV, we apply the theoretical framework to study cooperative and competitive dynamics, respectively. In these sections we report and discuss the phenomena resulting from the interaction of the diseases, thus recovering, under a single framework, the most important findings observed in previous works. In both cases, we check the validity of the theoretical predictions by comparing them with results obtained from extensive Monte Carlo simulations. Finally, we round off the work in Sec. V by giving some conclusions derived from our model and discussing about its implication for future research.

\section{THE MODEL}

We start by assuming that contagion processes are dictated by an unweighted and undirected contact network of $N$

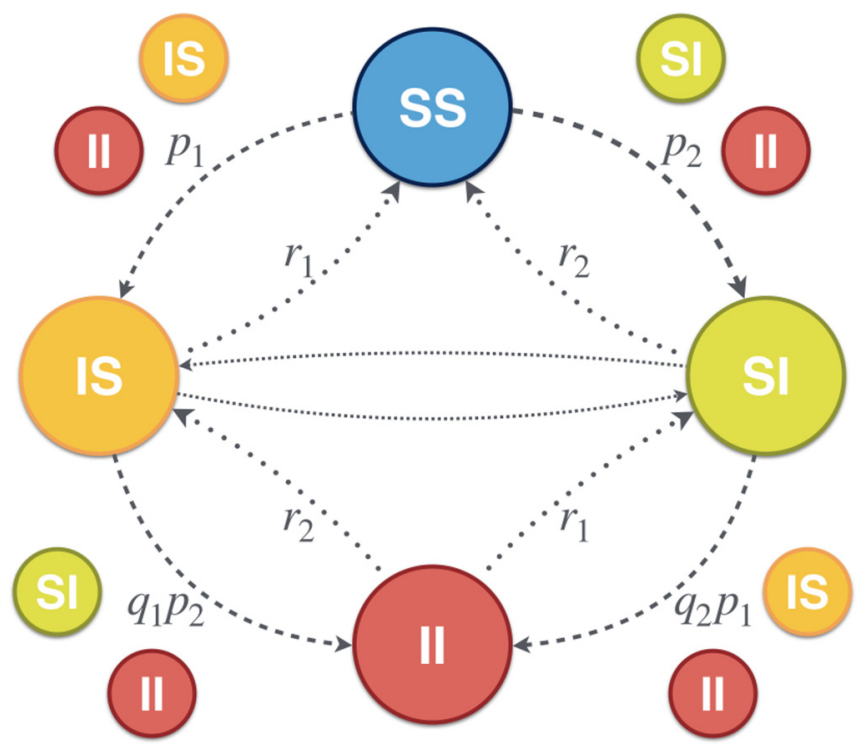

FIG. 1. Contagion and recovery microscopical processes considered in our model. Note that the contagion processes involving already infected individuals are influenced by the parameter $q$, which encodes the interaction between the two coexisting diseases.

nodes, each accounting for an agent, whereas interactions are determined by the $L$ network links. The network is described by its adjacency matrix $\mathbf{A}$ whose entries are defined as $A_{i j}=1$ if nodes $i$ and $j$ are connected and $A_{i j}=0$ otherwise. For the spreading dynamics, we consider that each disease $\beta$ can be individually modeled as a susceptible-infected-susceptible (SIS) dynamic, for which contagion and recovery probabilities are denoted as $p_{\beta}$ and $r_{\beta}$, respectively. In the absence of other pathogens, each disease $\beta$ spreads from an infected agent to a susceptible one with probability $p_{\beta}$, whereas infected agents become susceptible with probability $r_{\beta}$. Here, for the sake of simplicity, we will restrict to the case of two interacting diseases so that $\beta=1,2$. The interaction between the two diseases at work requires to couple two SIS dynamics. Therefore, agents subjected to a double SIS dynamics can be in four possible states which are: susceptible of contracting both diseases $(S S)$, infected by the first disease and susceptible of the second $(I S)$, susceptible of the first and infected of the second $(S I)$, and infected by both pathogens $(I I)$.

We now describe the transitions that govern the two coupled SIS dynamics, i.e., we define the transition probabilities between the former four epidemic states. In Fig. 1 we show all the microscopical processes involved in our model. First, for healthy (SS) agents we consider that the probability of being infected with pathogen $\beta$ is not affected by the presence of the other one. Therefore, both pathogens are transmitted to $S S$ agents with probabilities $p_{1}$ and $p_{2}$, respectively. To apply our model to mutually exclusive diseases, double contagions of fully susceptible agents are forbidden. The interaction between both circulating diseases is included via scaling parameters, $q_{1}\left(q_{2}\right)$, affecting the probability that an agent already infected by one disease catches the other one, as shown in Fig. 1 for the transitions $I S \rightarrow I I$ and $S I \rightarrow I I$. This way, $q_{\beta}<1$ implies that infected agents by one disease are less likely to get the other one, thus encoding a competition between both. However, $q_{\beta}>1$ means that contagions by 
one disease increase the vulnerability to the other one, which corresponds to a cooperative regime. Finally, all the recovery processes are assumed not to depend on the circulation of other pathogens, so that individuals overcome diseases 1 and 2 with probabilities $r_{1}$ and $r_{2}$, respectively. In this sense, let us remark that, in our model, $I I$ agents can recover from both diseases simultaneously and become $S S$ individuals. However, these flows are hardly observed. Therefore, the results reported below would not be significantly different from those obtained when double recovery events are forbidden.
Mathematically, our formalism contains a set of interdependent Markovian equations which enable to track the temporal evolution of the dynamical state of each agent $i$. Since there are four possible epidemic states for each of the $N$ agents, we require $3 N$ equations to completely characterize the evolution of the network. Given an agent, say $i$, let us denote as $\left[\rho^{\gamma}\right]_{i}^{t}$ the probability that this agent belongs to each of the following states $\gamma(\gamma=I S, S I, I I)$ at time $t$. Under the microscopical rules defined above, the temporal evolution of these probabilities reads as follows:

$$
\begin{gathered}
{\left[\rho^{I I}\right]_{i}^{t+1}=\left[\rho^{S I}\right]_{i}^{t}\left(1-r_{2}\right)\left\{1-\prod_{j}^{N}\left[1-A_{i j} p_{1} q_{2}\left(\left[\rho^{I S}\right]_{j}^{t}+\left[\rho^{I I}\right]_{j}^{t}\right)\right]\right\}} \\
+\left[\rho^{I S}\right]_{i}^{t}\left(1-r_{1}\right)\left\{1-\prod_{j}^{N}\left[1-A_{i j} p_{2} q_{1}\left(\left[\rho^{S I}\right]_{j}^{t}+\left[\rho^{I I}\right]_{j}^{t}\right)\right]\right\}+\left[\rho^{I I}\right]_{i}^{t}\left(1-r_{1}\right)\left(1-r_{2}\right), \\
{\left[\rho^{I S}\right]_{i}^{t+1}=\left[\rho^{S I}\right]_{i}^{t}\left(r_{2}\left\{1-\prod_{j}^{N}\left[1-A_{i j} p_{1} q_{2}\left(\left[\rho^{I S}\right]_{j}^{t}+\left[\rho^{I I}\right]_{j}^{t}\right)\right]\right\}\right)+\left[\rho^{I S}\right]_{i}^{t}\left(1-r_{1}\right) \prod_{j}^{N}\left[1-A_{i j} p_{2} q_{1}\left(\left[\rho^{S I}\right]_{j}^{t}+\left[\rho^{I I}\right]_{j}^{t}\right)\right]} \\
+\left[\rho^{I I}\right]_{i}^{t} r_{2}\left(1-r_{1}\right)+\left[\rho^{S S}\right]_{i}^{t}\left\{1-\prod_{j}^{N}\left[1-A_{i j}\left(p_{1}\left(\left[\rho^{I S}\right]_{j}^{t}+\left[\rho^{I I}\right]_{j}^{t}\right)+p_{2}\left(\left[\rho^{S I}\right]_{j}^{t}+\left[\rho^{I I}\right]_{j}^{t}\right)-p_{1} p_{2}\left(\left[\rho^{I I}\right]_{j}^{t}\right)^{2}\right)\right]\right\} f_{I S}, \\
{\left[\rho^{S I}\right]_{i}^{t+1}=\left[\rho^{I S}\right]_{i}^{t}\left(r_{1}\left\{1-\prod_{j}^{N}\left[1-A_{i j} p_{2} q_{1}\left(\left[\rho^{S I}\right]_{j}^{t}+\left[\rho^{I I}\right]_{j}^{t}\right)\right]\right\}\right)+\left[\rho^{S I}\right]_{i}^{t}\left(1-r_{2}\right) \prod_{j}^{N}\left[1-A_{i j} p_{1} q_{2}\left(\left[\rho^{I S}\right]_{j}^{t}+\left[\rho^{I I}\right]_{j}^{t}\right)\right]} \\
+\left[\rho^{I I}\right]_{i}^{t} r_{1}\left(1-r_{2}\right)+\left[\rho^{S S}\right]_{i}^{t}\left\{1-\prod_{j}^{N}\left[1-A_{i j}\left(p_{1}\left(\left[\rho^{I S}\right]_{j}^{t}+\left[\rho^{I I}\right]_{j}^{t}\right)+p_{2}\left(\left[\rho^{S I}\right]_{j}^{t}+\left[\rho^{I I}\right]_{j}^{t}\right)-p_{1} p_{2}\left(\left[\rho^{I I}\right]_{j}^{t}\right)^{2}\right)\right]\right\} f_{S I} .
\end{gathered}
$$

For the sake of readability, we have included the variable $\left[\rho^{S S}\right]_{i}^{t}$ whose value is automatically calculated as $\left[\rho^{S S}\right]_{i}^{t}=$ $1-\left[\rho^{I S}\right]_{i}^{t}-\left[\rho^{S I}\right]_{i}^{t}-\left[\rho^{I I}\right]_{i}^{t}$. Note that the contagion processes involving totally susceptible (SS) agents are shaped by $f_{I S}$ and $f_{S I}$. These factors account for the probability of contracting one disease when exposed to the other pathogen as well. To define this probability, we must define a rule for the case in which a fully susceptible agent is in contact with both pathogens when interacting with its neighbors. In this scenario, we assume that each disease will be contracted with the same probability. This way, the probabilities $f_{I S}$ and $f_{S I}$ read as

$$
\begin{aligned}
f_{I S} & =\frac{g_{I S}\left(1-0.5 g_{S I}\right)}{g_{I S}\left(1-0.5 g_{S I}\right)+g_{S I}\left(1-0.5 g_{I S}\right)}, \\
f_{S I} & =\frac{g_{S I}\left(1-0.5 g_{I S}\right)}{g_{I S}\left(1-0.5 g_{S I}\right)+g_{S I}\left(1-0.5 g_{I S}\right)},
\end{aligned}
$$

where $g_{I S}$ and $g_{S I}$ are the probabilities of making at least one infectious contact with individuals affected by the first and the second disease, respectively. These two probabilities can be expressed as

$$
\begin{aligned}
& g_{I S}=1-\prod_{j}^{N}\left[1-A_{i j} p_{1}\left(\left[\rho^{I S}\right]_{j}^{t}+\left[\rho^{I I}\right]_{j}^{t}\right)\right], \\
& g_{S I}=1-\prod_{j}^{N}\left[1-A_{i j} p_{2}\left(\left[\rho^{S I}\right]_{j}^{t}+\left[\rho^{I I}\right]_{j}^{t}\right)\right] .
\end{aligned}
$$

With these two equations we complete the Markovian description for two interacting diseases provided by Eqs. (1)-(3).

In the following sections we apply the formalism proposed above to study the impact of the interaction between simultaneous diseases. In particular, to put our focus on the effects of the interaction between diseases, we assume that both circulating pathogens, though different, are epidemiologically equivalent. Therefore, the only epidemiological parameters to be included in our framework are the infectivity $p=p_{1}=p_{2}$, the recovery rate, $r=r_{1}=r_{2}$, and the degree of interaction between pathogens, $q=q_{1}=q_{2}$. 

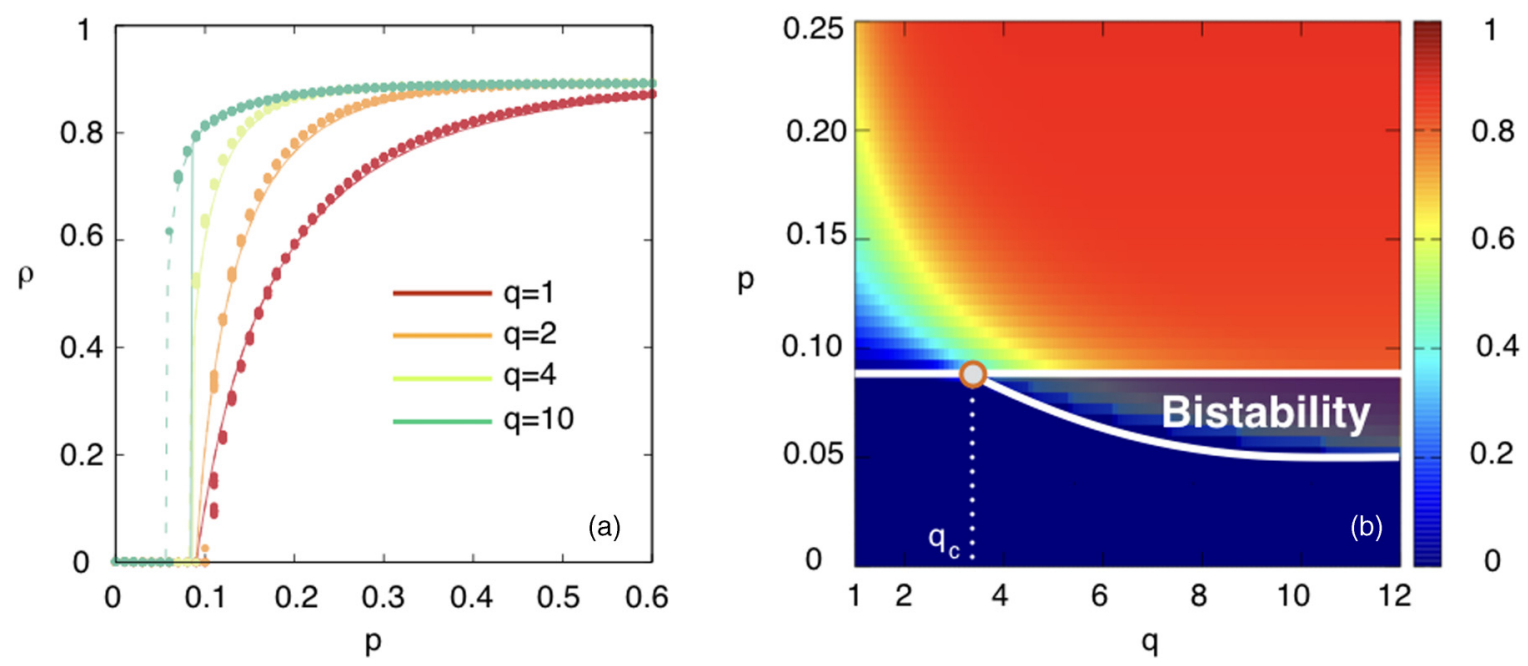

FIG. 2. Panel (a) shows the epidemic size $\rho$ as a function of the infectivity $p$ for several values of the interaction parameter $q$. The curves (solid and dashed) correspond to the results obtained by iterating Eqs. (1)-(3) when performing forward (solid) and backward (dashed) continuation schemes (see text for details). In its turn, points represent the results from 50 realizations of Monte Carlo simulations for each value of $p$. In panel (b) we plot the phase diagram $\rho(q, p)$ for the cooperative case. The solid white curves indicate the infectivity values for which the onset of epidemics takes place $\left(\rho>10^{-3}\right)$ in both backward and forward continuation branches. The critical point at $q_{c}$ pinpoints where the bistability (faded) region appears. In both panels we use an ER network of $N=1000$ nodes with $\langle k\rangle=8$, while $r=0.75$.

\section{COOPERATIVE DISEASES}

The spread of cooperative diseases can be captured in our formalism by setting $q \geqslant 1$ in Eqs.(1)-(3). In this case, we first explore the effects of increasing the cooperation strength. To do so, we study the dependence of the epidemic size on the infectivity $p$ and the interaction parameter $q$. For single diseases, the epidemic size is defined as the fraction of agents remaining infected when the epidemic has reached its stationary state. Following this definition, the epidemic size in the case of two diseases, denoted in the following as $\rho$, reads as

$$
\rho=\frac{1}{N} \sum_{i=1}^{N}\left(\rho_{i}^{I S}+\rho_{i}^{S I}+\rho_{i}^{I I}\right) .
$$

In Fig. 2(a) we represent the epidemic diagrams (curves) of an Erdös-Renyí (ER) network for several values of $q$ which range from the noninteracting case $(q=1)$ to that of large cooperation $(q=10)$. Similarly to the case of single noninteraction diseases, there is a threshold $p_{c}$ that separates the disease-free regime and the epidemic phase. Interestingly, the shape of the transition between both solutions strongly depends on the coupling $q$ between the two SIS dynamics. Specifically, for low values of the interaction between diseases, we observe the characteristic second-order transition of the single SIS model, i.e., at the epidemic threshold the absorbing state is no longer stable and the epidemic size $\rho$ grows smoothly as the infectivity increases. However, as the cooperation is strengthened, the continuous transition turns into a discontinuous one identified by a sharp variation of the epidemic size. This abrupt transition yields a bistable region for some $p$ values in which both the epidemic and the disease-free states are simultaneously stable. This bistability is manifested by making a forward and backward continuation of in $p$ when solving Eqs. (1)-(3) for a fixed value of $q$. The continuation method for the forward (backward) continuation branch solves Eqs. (1)-(3) by modifying adiabatically the value of the infectivity $p$. This way, the initial state for an infectivity value $p+\delta(p-\delta)$ is the steady configuration obtained for $p$ plus a small perturbation which is introduced to avoid remaining trapped in the absorbing state $(\rho=0)$ when the forward continuation branch is calculated. The solutions corresponding to the forward and backward continuations are plotted as solid and dashed curves, respectively.

To confirm the change in the nature of the epidemic onset observed from the solution of Eqs. (1)-(3) we perform simulations of the mechanistic model in which each agent possesses a particular dynamical state (SS, IS, SI, or SS), which is updated according to the contagion network and the microscopic rules defined in Sec. II. Figure 2(a) shows the results (points) obtained from 50 Monte Carlo simulations for each pair of $(p, q)$ values. From these results, it becomes clear that our model is able to reproduce very accurately the phenomena arisen from the cooperation between both diseases. In particular, for $q=10$, the Monte Carlo solutions clearly reveal that the transition becomes abrupt yielding the predicted bistable region in which both the epidemic and the disease-free states are simultaneously stable.

Once we have validated the framework, we can take advantage of the Markovian formalism to fully characterize the phase diagram in the $(p, q)$ space. In Fig. 2(b) we show the epidemic prevalence $\rho(q, p)$ and the curve $p_{c}(q)$ where epidemic onsets take place for each value of $q$. Interestingly, we can accurately identify the critical value $q_{c}$ for which this curve divides into two branches, signaling when bistability region (faded in the panel) shows up. This critical point $\left(q_{c}, p_{c}\left(q_{c}\right)\right)$ pinpoints the border between three phases: the epidemic, the disease-free, and the bistable ones.

Finally, we study the role played by the contact network on the abrupt onset of cooperative diseases. For this purpose, we fix $q=10$ and analyze the epidemic diagrams by tuning the degree heterogeneity of the underlying network using 


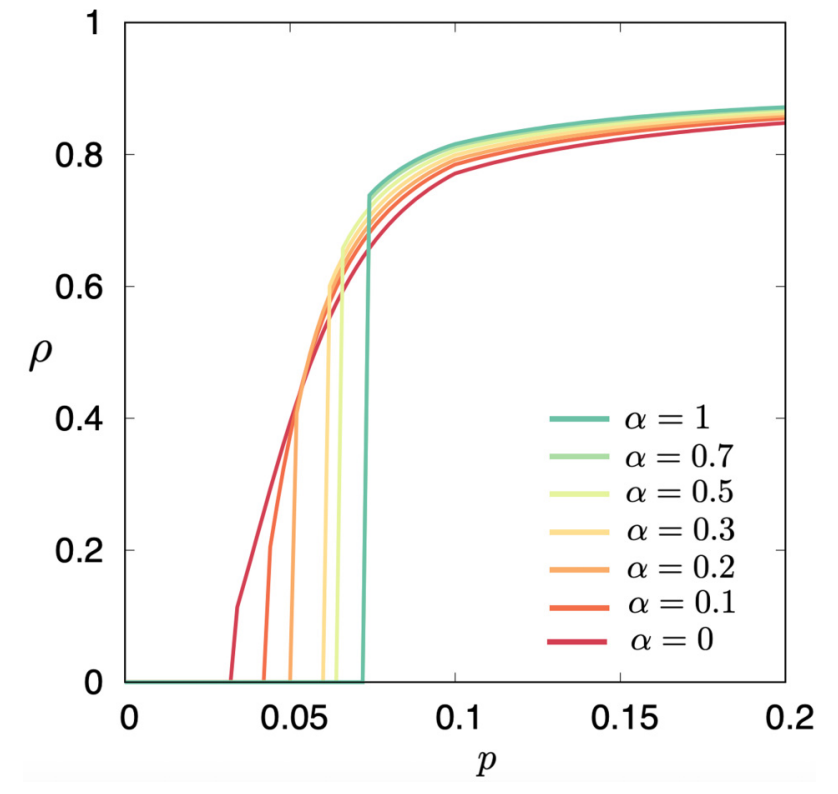

FIG. 3. Epidemic size computed following the forward continuation scheme as a function of the infectivity $p$ and the heterogeneity of the degree distribution of the underlying network governed by $\alpha$ (see text for more details). The recovery parameter has been set to $r=0.75$ whereas the interaction is fixed to $q=10$. Networks have $N=1000$ nodes and $\langle k\rangle=8$.

the model proposed in Ref. [60] (version A). In this model a network is constructed by mixing a fraction $\alpha$ of links randomly launched while the remaining $(1-\alpha)$ fraction of edges is set following a preferential attachment scheme. This way, by tuning parameter $\alpha$ this model allows to generate a uniparametric family of graphs that range from ER $(\alpha=1)$ and Barabási-Albert ( $\alpha=0$ ) networks, so the larger is $\alpha$ the more homogeneous is the network.

Figure 3 shows that, as expected, the epidemic threshold $p_{c}$ decreases as heterogeneity increases. However, homogeneous networks are less resilient to epidemic outbreaks than heterogeneous ones since the change in the epidemic size at the transition point becomes more pronounced as heterogeneity decreases. Thus, in cooperative scenarios, degree homogeneity, while delaying the epidemic onset, enhances the abrupt change in the epidemic prevalence at the critical point.

\section{COMPETITIVE DISEASES}

Once shown the formalism for the cooperative case, we now tackle the competition between two pathogens that propagate simultaneously. As a result of this competition, the infection probability of a pathogen decreases when affecting individuals who are already infected by the other one. Competitive interactions can be accomodated in Eqs. (1)-(3) by setting $q<1$. This choice includes a particular case that has drawn a lot of attention [53-56]: that of mutually exclusive pathogens. This particular scenario corresponds to the case $q=0$ in Eqs. (1)-(3) and captures the situation in which the infection by one pathogen generates cross-immunity to the other one, thus making state II inaccessible to agents.
The theoretical study of mutually exclusive pathogens has revealed the existence of different epidemic regimes depending on the value of the contagion rate $p$ and also on the initial partition of infected seeds for both diseases. According to these studies [53-56], the outcome of fully competitive disease ranges from the dominance of one single disease (and the resulting extinction of the other one) to a regime of coexistence in which the whole epidemic prevalence contains infected individuals of both diseases.

To study this phenomenology in the Markovian framework we set, as anticipated above, $q=0$ and study the impact of both diseases as a function of the contagion rate $p$. Regarding the initial infectious seeds, we bias the initial configuration toward one of the pathogens by infecting $2 \%$ of the population with the first disease and $1 \%$ with the second one. Starting from this setup we iterate Eqs. (1)-(3) and compute the global epidemic size $\rho$ together with the difference between the prevalences of each disease, $\left|\rho^{I S}-\rho^{S I}\right|$, where

$$
\begin{aligned}
& \rho^{I S}=\frac{1}{N} \sum_{i=1}^{N} \rho_{i}^{I S}, \\
& \rho^{S I}=\frac{1}{N} \sum_{i=1}^{N} \rho_{i}^{S I} .
\end{aligned}
$$

With these two order parameters we are able to distinguish between the case of full dominance of one disease $(\rho=$ $\left.\left|\rho^{I S}-\rho^{S I}\right|>0\right)$ and that corresponding to equal prevalence $\left(\rho>0\right.$ and $\left.\left|\rho^{I S}-\rho^{S I}\right|=0\right)$.

In Fig. 4 we monitor these two order parameters as a function of the infection probability $p$. From this plot, it becomes clear that the Markovian framework reproduces the phenomenology previously reported for competitive diseases. Namely, for $p<p_{c}$, the absorbing disease-free state is the only stable solution. Note that, being the infection probabilities of both pathogens the same, this epidemic threshold is exactly the same as for independent SIS diseases. Therefore, $p_{c}$ is given by $[14,59]$

$$
p_{c}=\frac{r}{\Lambda_{\max }(\mathbf{A})},
$$

where $\Lambda_{\max }(\mathbf{A})$ is the maximum eigenvalue of the adjacency matrix A. Above this threshold, $p>p_{c}$ the global epidemic size grows smoothly as the infectivity $p$ increases. However, two different behaviors show up. For $p>p_{c}$ we find a dominance regime in which one disease (here the first one) prevails over the other one (whose prevalence is zero). In particular, the dominant disease is that with the larger initial proportion of infected individuals (here the first one). However, the dominance regime suddenly breaks up when the infectivity reaches a second threshold, denoted in the following as $p_{c}^{\prime}$. For $p>p_{c}^{\prime}$, the second pathogen no longer disappears and the steady state now comprises an equal prevalence of both diseases. Thus, the coexistence regime appears when $p>p_{c}^{\prime}$. These results [obtained by solving Eqs. (1)-(3) with $q=0$ ] are totally in agreement with those obtained from Monte Carlo simulations of the mutually exclusive coupled SIS model, reported as points in Fig. 4.

It is possible to explain the full dominance regime in physical terms by recalling that once $p \gtrsim p_{c}$ the most 


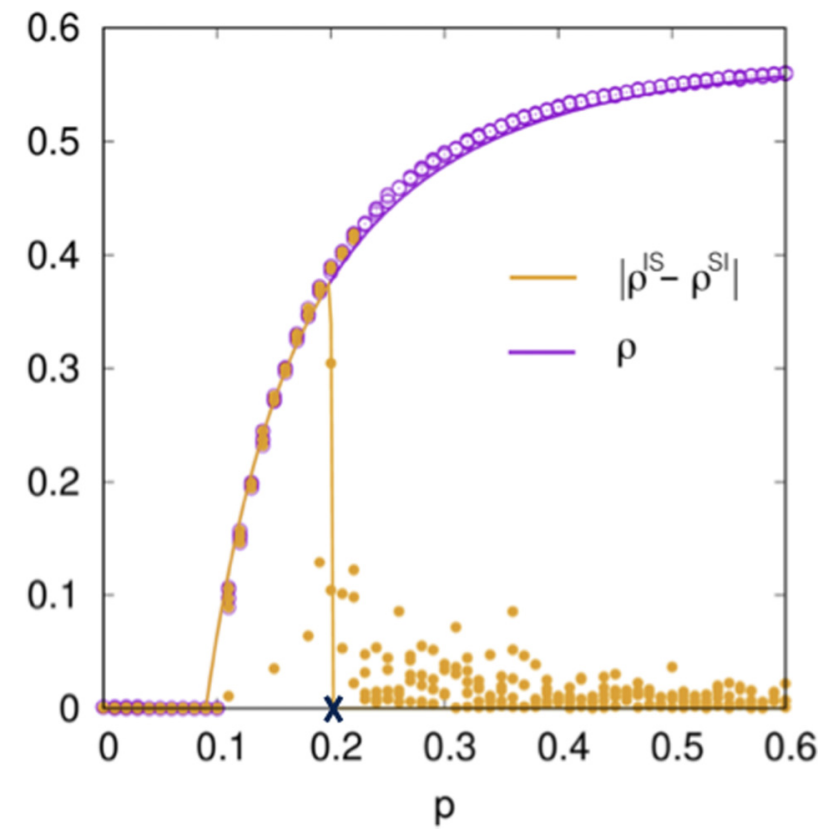

FIG. 4. Evolution of the global epidemic size $\rho$ (purple empty points) and the difference between the prevalences of both diseases (orange solid points) as a function of the infectivity $p$ in the fully competitive case $q=0$. These points are the results obtained from 50 realizations of Monte Carlo simulations for each value of $p$. In its turn, solid lines denote the solution obtained by iterating Eqs. (1)-(3). Finally, the cross shows the theoretical estimation of the threshold $p_{c}^{\prime}$ as obtained from Eq. (18). The network and the recovery probability are the same as those used in Fig. 2.

abundant pathogen blocks, due to the cross-immunization effect, many contagion pathways for the minority one. This way, the effective network that remains for the spread of the minority pathogen has an effective epidemic threshold larger than the original one, $p_{c}$, thus preventing its dissemination for $p \gtrsim p_{c}$. However, as $p$ increases the initial unbalanced configuration loses its relevance and the transmission of both diseases become identical, thus leading to a coexistence regime. This latter phase of the dynamics shows up when the second threshold is exceeded, $p>p_{c}^{\prime}$.

Let us now take advantage of the validity of the Markovian formalism to derive some analytical calculations about the second threshold, $p_{c}^{\prime}$, separating the full dominance and the coexistence regimes in the case of mutually exclusive diseases $(q=0)$. We start by making the following change of variables:

$$
\begin{aligned}
& \rho_{i}^{t}=\left[\rho^{I S}\right]_{i}^{t}+\left[\rho^{S I}\right]_{i}^{t}, \\
& \Delta_{i}^{t}=\left[\rho^{I S}\right]_{i}^{t}-\left[\rho^{S I}\right]_{i}^{t} .
\end{aligned}
$$

Note that this change leaves the third set of variables $\left[\rho^{I I}\right]_{i}^{t}$ unaltered since for the case $q=0$ we have $\left[\rho^{I I}\right]_{i}^{t}=0 \forall i$. From Eqs. (1)-(2) we can write the Markovian equations governing the time evolution of the new variables as

$$
\begin{gathered}
\rho_{i}^{t+1}=(1-r) \rho_{i}^{t}+\left(1-\rho_{i}^{t}\right) q_{i}\left(\vec{\rho}^{t}\right), \\
\Delta_{i}^{t+1}=(1-r) \Delta_{i}^{t}+\left(1-\rho_{i}^{t}\right) q_{i}\left(\vec{\rho}^{t}\right)\left(f^{I S}-f^{S I}\right),
\end{gathered}
$$

where $f^{I S}=f^{I S}\left(\vec{\rho}^{t}, \vec{\Delta}^{t}\right)$ and $f^{S I}=f^{S I}\left(\vec{\rho}^{t}, \vec{\Delta}^{t}\right)$, and we have defined

$$
q_{i}^{t}\left(\vec{\rho}^{t}\right)=\left[1-\prod_{j=1}^{N}\left(1-p A_{i j} \rho_{j}^{t}\right)\right],
$$

for the sake of clarity. Note that the first equation, Eq. (14), is formally identical to that of an ordinary SIS model. This implies that, either in the case that one pathogen dominates over the other or in the regime in which they coexist, the overall prevalence is the same as that of one single pathogen spreading in the network. Thus, the form of Eq. (14) pinpoints that, to capture the second transition point $p_{c}^{\prime}$, we should focus on the behavior of $\left\{\Delta_{i}^{t}\right\}$, Eq. (15), since in the full dominance phase $\left(p_{c}<p<p_{c}^{\prime}\right)$ we have $\left\{\Delta_{i}^{t}\right\}=\left\{\rho_{i}^{t}\right\}$, whereas the coexistence phase $\left(p>p_{c}^{\prime}\right)$ is characterized by $\left\{\Delta_{i}^{t}\right\}=\overrightarrow{0}$.

Let us consider that the dynamics is in its stationary regime, i.e., $\rho_{i}^{t+1}=\rho_{i}^{t}=\rho_{i}^{*}$ and $\Delta_{i}^{t+1}=\Delta_{i}^{t}=\Delta_{i}^{*} \forall i$. In this case, Eq. (15) becomes

$$
\Delta_{i}^{*}=\rho_{i}^{*}\left[f^{I S}\left(\vec{\rho}^{*}, \vec{\Delta}^{*}\right)-f^{S I}\left(\vec{\rho}^{*}, \vec{\Delta}^{*}\right)\right] .
$$

Since we are interested in capturing the transition between $\overrightarrow{\Delta^{*}}=0$ and $\vec{\Delta}^{*} \neq 0$ we consider that the values $\Delta_{i}^{*}$ are small, $\Delta_{i}^{*}=\epsilon_{i}^{*}$. This allows us to linearize Eq. (17), and obtain the equation that has to be fulfilled at $p=p_{c}^{\prime}$ :

$$
\begin{aligned}
& \epsilon_{i}^{*}=\sum_{l=1}^{N}\left\{\rho_{i}^{*} \frac{p_{c}^{\prime} A_{i l} q_{i}\left(\vec{\rho}^{*} / 2\right)}{\left(1-p_{c}^{\prime} A_{i l} \rho_{l}^{*} / 2\right)\left[1-q_{i}\left(\vec{\rho}^{*} / 2\right)^{2}\right]}\right\} \epsilon_{l}^{*} \\
& =\sum_{l=1}^{N} \mathcal{M}_{i l}\left(\vec{\rho}^{*} ; p_{c}^{\prime}\right) \epsilon_{l}^{*} \text {. }
\end{aligned}
$$

Thus, to find the value $p_{c}^{\prime}$ one needs to find the minimum value of $p$ that fulfills that matrix $\mathcal{M}$ has 1 as eigenvalue. In practical terms, since $\mathcal{M}$ depends on the overall prevalence, $\vec{\rho}^{*}(p)$, one should first solve the SIS diagram $\vec{\rho}^{*}(p)$ for a single disease and, by inserting the resulting stationary values $\vec{\rho} *(p)$ in matrix $\mathcal{M}$, identify the value $p_{c}^{\prime}$ that fulfils Eq. (18).

Following Eq. (18) we obtained the estimation of $p_{c}^{\prime}$ for the ER graphs used in our numerical simulations so far. The result (see the cross in Fig. 4) reveals the accuracy of the theoretical prediction. We can therefore use this result to analyze, without the need of solving Eqs. (1)-(2), the two thresholds, $p_{c}$ and $p_{c}^{\prime}$, for any given network. To understand the role of network topology on the coexistence of the two diseases, we have explored the evolution of these two thresholds when varying the degree heterogeneity of the underlying network. To this end we make use of the interpolating model network [60] and vary the parameter $\alpha \in[0,1]$ governing the formation of the network. In Fig. 5 we show the curves $p_{c}(\alpha)$ and $p_{c}^{\prime}(\alpha)$ as obtained from Eqs. (11) and (18), respectively. As a result we obtain the phase diagram in the $(\alpha, p)$ space showing the limits between the three phases. Interestingly, the second threshold $p_{c}^{\prime}$ follows a decreasing trend as heterogeneity increases $\left(\alpha \rightarrow 0^{+}\right)$similar to the well-known behavior of the epidemic threshold $p_{c}$.

Finally, we use the Markovian framework, Eqs. (1)-(3), to explore less restrictive competitive scenarios in which $q \neq 0$. This way, in Fig. 6 we study the dependency of the second 


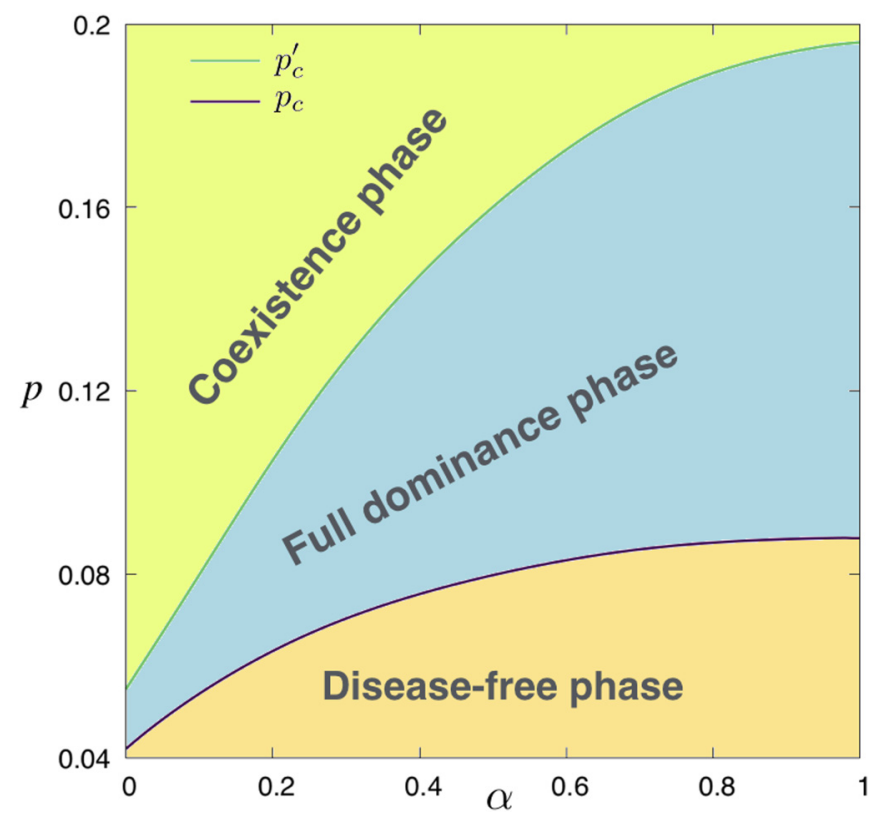

FIG. 5. Phase diagram of the fully competitive model $(q=0)$ in the $(\alpha, p)$ space. Parameter $\alpha$ governs the degree heterogeneity of the underlying contact network (see main text). This diagram is obtained by calculating the curves $p_{c}(\alpha)$ and $p_{c}^{\prime}(\alpha)$ obtained from Eqs. (11) and (18), respectively. These curves pinpoint the borders between the three possible regimes as reported in the plot and show that the second threshold $p_{c}^{\prime}$ increases as homogeneity $(\alpha)$ increases, as already known for the first (epidemic) threshold $p_{c}$. The recovery rate has been fixed to $r=0.75$. Networks have $N=1000$ nodes and $\langle k\rangle=8$.

epidemic threshold $p_{c}^{\prime}$ on the interaction parameter $q$ for several values of the recovery rate $r$ and the average degree of the ER contact network $\langle k\rangle$. In both panels of Fig. 6, it becomes clear that increasing $q$ from the fully-competitive case makes the full-dominance regime more vulnerable. Note, however, that, even when the two pathogens are not mutually exclusive from a microscopical point of view $(q \neq 0)$, the existence of a negative interaction between them can lead to the vanishing of the weak disease in the macroscopic state. Obviously, as $q$ increases, this competition is softened and the second threshold $p_{c}^{\prime}$ approaches the first one $p_{c}$ (dotted lines in Fig. 6). When $p_{c}^{\prime}=p_{c}$ the full dominance phase no longer shows up. Interestingly, this effect strongly depends on the value of the recovery rate and the average degree. In particular, Fig. 6(a) reveals that the larger is the recovery rate the more resilient is the full dominance regime. This can be explained by noticing that increasing the recovery rate reduces the formation of large clusters of the weak disease, thus increasing the dynamical advantage of the dominant one. In its turn, in Fig. 6(b) we report that increasing the average degree of the network (while keeping constant the recovery rate) favors the emergence of the coexistence regime. To explain this, let us remark that the extinction of one disease occurs since the other one blocks many of its spreading pathways. Thus, a larger average degree promotes more potential contagion routes, making the full dominance regime more vulnerable.

\section{CONCLUSIONS}

Phenomena such as the existence of simultaneous outbreaks or the extinction of some infectious strains due to the presence of other ones demand the incorporation of the interaction between different pathogens in epidemic models. The most relevant contribution of this work is to provide a versatile Markovian framework capable of capturing different types of interaction between coexisting diseases. Besides, this framework accounts for the whole structure of connections of the underlying contact network, thus abandoning the assumptions about the statistical equivalence of nodes within the same degree class. The validity of the proposed equations has been tested by comparing with the results obtained from Monte Carlo simulations, showing an excellent agreement for any degree of epidemic prevalence.
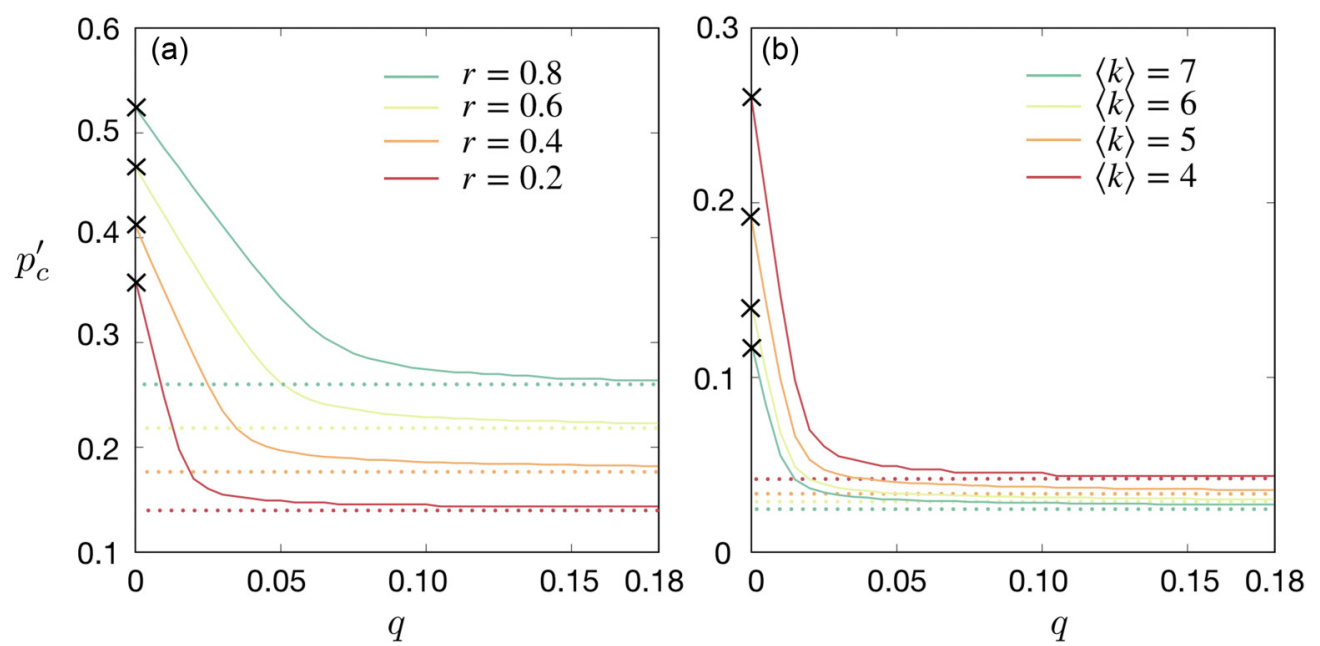

FIG. 6. In this figure we show the second epidemic threshold $p_{c}^{\prime}$ as a function of the interaction parameter $q$ for several values of the recovery rate $r$ (a) and different average degree of the underlying ER network (b). In (a) the average degree has been set to $\langle k\rangle=4$, whereas in panel (b) the recovery rate is fixed to $r=0.2$. In both panels the crosses account for the theoretical estimation of $p_{c}^{\prime}$ in the fully competitive case $(q=0)$ according to Eq. (18). Dotted lines indicate the value of the threshold $p_{c}$ obtained from Eq. (11) for each $(r,\langle k\rangle)$. 
Supported by the validity of the Markovian equations, we have explored the role that the interaction between diseases plays on their unfolding. For cooperative diseases, we have shown that, as the interaction between pathogens increases, the smooth transition at the epidemic threshold turns into a first-order one in which there is an abrupt transition between the absorbing (healthy) state and the epidemic one. We have used the formalism to compute the critical value for the degree of cooperation, $q_{c}$, that triggers the abrupt transition. In its turn, for competitive diseases we have analyzed the case of mutually exclusive pathogens. In this case we have shown that the epidemic phase is divided into two different regimes: the full dominance phase (in which only one pathogen spreads across the network while the other disappears) and the coexistence phase (in which both pathogens spread simultaneously). To shed more light into this phenomenon, we have linearized the Markovian equation to derive an analytical estimation of the infectivity threshold, $p_{c}^{\prime}$, that separates both phases.

Apart from recovering the phenomenology reported in the literature for both competitive and cooperative scenarios, the use of the MMCA enables to address the impact of the network structure on the emergence of these phenomena. In particular, we have revealed that, in the cooperative case, the first-order transition is less abrupt for heterogeneous topologies, for it only affects the most connected nodes and their neighborhood. However, the heterogeneity of the underlying contact network favors the coexistence of competing diseases, since the existence of more available routes decreases the confluence of both pathogens.

Finally, although it is not explicitly studied in the manuscript, few words should be spent on how interaction also shapes the extinction probability and mean lifetime of diseases since, on finite networks, the endemic state is only metastable and every disease will eventually die due to stochastic fluctuations $[61,62]$. On the one hand, for cooperative diseases, the interaction between both pathogens fosters their propagation over the network, leading to a higher incidence and extremely long extinction times. This boost is specially relevant in the early stages of the diseases, for it clearly reduces the probability of remaining trapped in the absorbing state. On the other hand, the competition between two diseases blocks many of the available spreading routes for each pathogen. This way, each disease propagates over an effective network with smaller average degree, thus increasing their extinction probabilities and making the metastable endemic state more vulnerable.

In a nutshell, the Markovian benchmark presented here has allowed a systematic study of different degrees of positive and negative interactions between the pathogens and the accurate characterization of the corresponding phase diagrams. The analytical derivation of the infection threshold between fulldominance and coxistence regime for any arbitrary network opens the door to the design of containment measures via the introduction of highly infective but innocuous computer viruses aimed at decreasing the damage of malware [63]. Finally, our formalism paves the way for the study of more sophisticated interacting spreading processes such as complex social contagions [64-67] applied, for instance, to the competition of ideas [68] or innovations [69].

\section{ACKNOWLEDGMENTS}

We acknowledge discussions, comments, and useful insights from Y. Moreno. D.S.-P. acknowledges financial support from Gobierno de Aragón through a doctoral fellowship. J.G.-G and D.S.-P. acknowledge support from MINECO and FEDER funds (Grants No. FIS2015-71582-C2 and No. FIS2017-87519-P), and Gobierno de Aragón/Fondo Europeo de Desarrollo Regional (FEDER) (Grant No. E36_17R to FENOL group). F.G. acknowledges partial support by the Deutsche Forschungsgemeinschaft (DFG) under the grant GH 176/1-1 (idonateproject No. 345463468). S.M. acknowledges partial funding from the Spanish Ministry of Science, Innovation and Universities, the National Agency for Research Funding AEI and FEDER (EU) under the grant PACSS (RTI2018-093732-B-C22) and the Maria de Maeztu program for Units of Excellence in R\&D (MDM-2017-0711).
[1] World Health Organization, The World Health Report (2016).

[2] R. M. Anderson, R. M. May, and B. Anderson, Infectious Diseases of Humans: Dynamics and Control (Oxford University Press, Oxford, 1992).

[3] M. J. Keeling and P. Rohani, Modeling Infectious Diseases in Humans and Animals. (Princeton University Press, Princeton, NJ, 2008).

[4] H. W. Hethcote, The mathematics of infectious diseases, SIAM Rev. 42, 599 (2000).

[5] D. J. Daley and J. Gani, Epidemic Modelling (Cambridge University Press, Cambridge, UK, 1999).

[6] R. Pastor-Satorras, C. Castellano, P. Van Mieghem, and A. Vespignani, Epidemic processes in complex networks, Rev. Mod. Phys. 87, 925 (2015).

[7] W. Wang, M. Tang, H. E. Stanley, and L. A. Braunstein, Unification of theoretical approaches for epidemic spreading on complex networks, Rep. Prog. Phys. 80, 036603 (2017).
[8] A. L. Lloyd, and R. M. May, How viruses spread among computers and people, Science 292, 1316 (2001).

[9] L. J. S. Allen, Some Discrete-Time SI, SIR, and SIS Epidemic Models, Math. Biosciences 124, 83 (1994).

[10] R. Albert and A.-L. Barabasi, Statistical mechanics of complex networks, Rev. Mod. Phys. 74, 47 (2002).

[11] M. E. J. Newman, The structure and function of complex networks, SIAM Rev. 45, 167 (2003)

[12] S. Boccaletti, V. Latora, Y. Moreno, M. Chavez, and D.-U. Hwang, Complex networks: structure and dynamics, Phys. Rep. 424, 175 (2006).

[13] R. Pastor-Satorras and A. Vespignani, Epidemic Spreading in Scale-Free Networks, Phys. Rev. Lett. 86, 3200 (2001).

[14] B. Guerra and J. Gómez-Gardeñes, Annealed and mean-field formulations of disease dynamics on static and adaptive networks, Phys. Rev. E 82, 035101 (2010). 
[15] C. Castellano and R. Pastor-Satorras, Thresholds for Epidemic Spreading in Networks, Phys. Rev. Lett. 105, 218701 (2010).

[16] A. V. Goltsev, S. N. Dorogovtsev, J. G. Oliveira, and J. F. F. Mendes, Localization and Spreading of Diseases in Complex Networks, Phys. Rev. Lett. 109, 128702 (2012).

[17] M. De Domenico, A. Solé-Ribalta, E. Cozzo, M. Kivelä, Y. Moreno, M. A. Porter, S. Gomez, and A. Arenas, Mathematical Formulation of Multi-Layer Networks, Phys. Rev. X 3, 041022 (2013).

[18] S. Boccaletti et al., The structure and dynamics of multilayer networks, Phys. Rep. 544, 1 (2014).

[19] M. Kivelä et al., J. Complex Netw. 2, 203 (2014).

[20] N. Masuda and R. Lambiotte, A Guide to Temporal Networks (World Scientific, Singapore, 2016).

[21] L. Speidel, K. Klemm, V. M. Eguíluz, and N. Masuda, Temporal interactions facilitate endemicity in the susceptible-infectedsusceptible epidemic model, New J. Phys. 18, 073013 (2016).

[22] N. Masuda and P. Holme (eds.), Temporal Network Epidemiology (Springer, Singapore, 2017).

[23] E. Valdano, M. Re Fiorentin, Ch. Poletto, and V. Colizza, Epidemic Threshold in Continuous-Time Evolving Networks, Phys. Rev. Lett. 120, 068302 (2018).

[24] M. Ogura, V. M. Preciado, and N. Masuda, Optimal containment of epidemics over temporal activity-driven networks, SIAM J. Appl. Math. 79, 986 (2019).

[25] V. Colizza and A. Vespignani, Invasion Threshold in Heterogeneous Metapopulation Networks, Phys. Rev. Lett. 99, 148701 (2007).

[26] V. Colizza, R. Pastor-Satorras, and A. Vespignani, Reactiondiffusion processes and metapopulation models in heterogeneous networks, Nat. Phys. 3, 276 (2007).

[27] D. Balcan, V. Colizza, B. Gonçalves, H. Hu, J. J. Ramasco, and A. Vespignani, Multiscale Mobility networks and the spatial spreading of infectious diseases, Proc. Natl. Acad. Sci. USA 106, 21484 (2009).

[28] D. Balcan and A. Vespignani, Phase transitions in contagion processes mediated by recurrent mobility patterns, Nat. Phys. 7, 581 (2011).

[29] V. Belik, T. Geisel, and D. Brockmann, Natural Human Mobility Patterns and Spatial Spread of Infectious Diseases, Phys. Rev. X 1, 011001 (2011).

[30] E. Cozzo, R. A. Baños, S. Meloni, and Y. Moreno, Contactbased social contagion in multiplex networks, Phys. Rev. E 88, 050801(R) (2013).

[31] G. F. de Arruda, E. Cozzo, T. P. Peixoto, F. A. Rodrigues, and Y. Moreno, Disease Localization in Multilayer Networks, Phys. Rev. X 7, 011014 (2017).

[32] D. Soriano-Paños, A. Arenas, and J. Gómez-Gardeñes, Spreading processes in Multiplex Metapopulations Containing Different Mobility Networks, Phys. Rev. X 8, 031039 (2018).

[33] S. Funk, M. Salathé, and V. A. A. Jansen, Modelling the influence of human behavior on the spread of infectious diseases: A review, J. R. Soc. Interface 7, 1247 (2010).

[34] C. T. Bauch and A. P. Galvani, Social factors in epidemiology, Science 342, 47 (2013).

[35] A. Cardillo, C. Reyes-Suárez, F. Naranjo, and J. GómezGardeñes, The evolutionary vaccination dilemma in complex networks, Phys. Rev. E 88, 032803 (2013).

[36] B. Steinegger et al., Interplay between cost and benefits triggers nontrivial vaccination uptake, Phys. Rev. E 97, 032308 (2018).
[37] J. Gómez-Gardeñes, D. Soriano-Paños, and A. Arenas, Critical regimes driven by recurrent mobility patterns of reactiondiffusion processes in networks, Nat. Phys. 14, 391 (2018).

[38] M. J. Keeling and C. A. Gilligan, Metapopulation dynamics of bubonic plague, Nature 407, 903 (2000).

[39] C. Granell and P. J. Mucha, Epidemic spreading in localized environments with recurrent mobility patterns, Phys. Rev. E 97, 052302 (2018).

[40] R. Acuna-Soto, C. Viboud, and G. Chowell, Influenza and pneumonia mortality in 66 large cities in the United States in years surrounding the 1918 pandemic, PLOS One 6, e23467 (2011).

[41] www.who.int/hiv/topics/tb/about_tb/en.

[42] N. G. Reich et al. Interactions between serotypes of dengue highlight epidemiological impact of cross-immunity, J. R. Soc. Interface 10, 20130414 (2013).

[43] I. Rodriguez-Barraquer et al., Impact of preexisting dengue immunity on Zika virus emergence in a dengue endemic region, Science 363, 607 (2019).

[44] R. G. Webster, W. J. Bean, O. T. Gorman, T. M. Chambers, and Y. Kawaoka, Evolution and ecology of influenza A viruses, Micorbiol. Mol. Biol. Rev. 56, 152 (1992).

[45] S. M. Asaduzzaman, J. Ma, and P. van den Driessche, Estimation of cross-immunity between drifted strains of influenza A/H3N2, Bull. Math. Biol. 80, 657 (2018).

[46] J. Sanz, Ch.-Y. Xia, S. Meloni, and Y. Moreno, Dynamics of Interacting Diseases, Phys. Rev. X 4, 041005 (2014).

[47] N. Azimi-Tafreshi, Cooperative epidemics on multiplex networks, Phys. Rev. E 93, 042303 (2016).

[48] W. Cai, L. Chen, F. Ghanbarnejad, and P. Grassberger, Avalanche outbreaks emerging in cooperative contagions, Nat. Phys. 11, 936 (2015).

[49] L. Hébert-Dufresne and B. M. Althouse, Complex dynamics of synergistic coinfections on realistically clustered networks, Proc. Nat. Acad. Sci. USA 112, 10551 (2015).

[50] L. Chen, F. Ghanbarnejad, and D. Brockmann, Fundamental properties of cooperation contagion processes, New J. Phys. 19, 103041 (2017).

[51] P.-B. Cui, F. Colaiori, and C. Castellano, Mutually cooperative epidemics on power-law network, Phys. Rev. E 96, 022301 (2017).

[52] P.-B. Cui, F. Colaiori, and C. Castellano, Effect of network clustering on mutually cooperative coinfections, Phys. Rev. E 99, 022301 (2019).

[53] F. D. Sahneh and C. Scoglio, Competitive epidemic spreading over arbitrary multilayer networks, Phys. Rev. E 89, 062817 (2014).

[54] B. Karrer and M. E. J. Newman, Competing epidemics on complex networks, Phys. Rev. E 84, 036106 (2011).

[55] O. Kogan et al., Two-strain competition in quasineutral stochastic disease dynamics, Phys. Rev. E 90, 042149 (2014).

[56] C. Poletto et al., Characterising two-pathogen competition in spatially structured environments, Sci. Rep. 5, 7895 (2015).

[57] F. Pinotti, E. Fleury, D. Guillemot, P.-Y. Boelle, and C. Poletto, Host contact dynamics shapes richness and dominance of pathogen strains, PLoS Comput. Biol. 15, e1006530 (2019).

[58] W. Wang, Q.-H. Liu, J. Liang, Y. Hu, and T. Zhou, Coevolution spreading in complex networks, Phys. Rep. 820, 1 (2019).

[59] S. Gómez, A. Arenas, J. Borge-Holthoefer, S. Meloni, and Y. Moreno, Discrete-time Markov chain approach to contact based 
diseases spreading in complex networks, Europhys. Lett. 89, 38009 (2010).

[60] J. Gómez-Gardeñes and Y. Moreno, From scale-free to ErdösRényi networks, Phys. Rev. E 73, 056124 (2006).

[61] J. Hindes and I. B. Schwartz, Epidemic Extinction and Control in Heterogeneous Networks, Phys. Rev. Lett. 117, 028302 (2016).

[62] J. Hindes and I. B. Schwartz, Epidemic extinction paths in complex networks, Phys. Rev. E 95, 052317 (2017).

[63] V. Bontchev, Are "Good" Computer Viruses Still a Bad Idea? Proceedings EICAR (1994), pp. 25-47.

[64] M. Granovetter, Threshold models of collective behavior, Am. J. Sociol. 83, 1420 (1978).
[65] D. J. Watts, A simple model of global cascades on random networks, Proc. Natl. Acad. Sci. USA 99, 5766 (2002).

[66] J. Gómez-Gardeñes, L. Lotero, S. N. Taraskin, and F. J. PérezReche, Explosive Contagion in Networks, Sci. Rep. 6, 19767 (2016).

[67] I. Iacopini, G. Petri, A. Barrat, and V. Latora, Simplicial models of social contagion, Nat. Commun. 10, 2485 (2019).

[68] Y. Wang, G. Xiu, and J. Liu, Dynamics of competing ideas in complex social systems, New J. Phys. 14, 013015 (2012).

[69] B. Min and M. San Miguel, Competition and dual users in complex contagion processes, Sci. Rep. 8, 14580 (2018). 